\title{
Actin turnover protects the cytokinetic contractile ring from structural instability
}

\author{
Zachary McDargh ${ }^{1}$, Tianyi Zhu ${ }^{1}$, Hongkang Zhu ${ }^{1}$ and Ben O’Shaughnessy ${ }^{1, *}$ \\ ${ }^{1}$ Department of Chemical Engineering, Columbia University, New York, NY 10027, USA \\ *To whom correspondence may be addressed. Email: bo8@columbia.edu.
}

Keywords: cytokinesis, contractile ring, $\mathrm{ADF} /$ cofilin, actin turnover, instability 


\begin{abstract}
In common with other actomyosin contractile cellular machineries, actin turnover is required for normal function of the cytokinetic contractile ring. Cofilin is an actin-binding protein contributing to actin turnover by severing actin filaments, required for cytokinesis by many organisms. In fission yeast cofilin mutants, contractile rings exhibit bridging instabilities when actin bundles peel from the plasma membrane into straight bridges, but their origin is unclear. Here we used molecularly explicit molecular simulations of the fission yeast contractile ring to examine the role of cofilin. Simulations reproduced the $\sim 3$ observed cycles of bridging and reassembly during constriction, each lasting $\sim 6$ mins. Bridging occurred in ring segments with low myosin II Myo2 density, as observed. Lack of cofilin severing produced $\sim 2$-fold longer filaments and, consequently, $\sim 2$-fold higher ring tensions. Simulations identified bridging as originating in the boosted ring tension, which increased centripetal forces pulling actin from the membrane against Myo2, the primary anchor of actin. Thus, cofilin serves a critical role in cytokinesis by protecting the contractile ring against bridging, the principal structural threat to the cytokinetic ring.
\end{abstract}

\title{
Summary statement:
}

Molecularly explicit simulations showed that cofilin-mediated actin severing protects the fission yeast cytokinetic contractile ring from instabilities where actin peels away into straight bridges. 


\section{Introduction}

Turnover of actin and other components is a universal feature of actomyosin contractile machineries in cells. Actin turns over through nucleation of filamentous actin by formins, Arp2/3 or other factors (Pollard, 2007), and by dissociation and/or disassembly of filaments. Cofilin is an actin-binding protein whose severing activity is critical for actin turnover in many machineries, including the actomyosin contractile ring that drives cell division during cytokinesis (D'Avino et al., 2015; Pollard and O'Shaughnessy, 2019; Pollard and Wu, 2010a). Cofilin is required for cytokinesis in many organisms (Abe et al., 1996; Gunsalus et al., 1995; Ono et al., 2003) including fission yeast (Nakano and Mabuchi, 2006), but many aspects of the mechanisms that make cofilin essential to cytokinesis remain poorly understood.

Fission yeast assembles a contractile ring from several hundred precursor protein complexes called nodes, each node incorporating myosin II Myo2, formin Cdc12 that nucleates and grows actin filaments, and other components (Wu et al., 2006). Normal ring assembly requires cofilin, as shown by studies of cytokinesis with mutants (Chen and Pollard, 2011; Nakano and Mabuchi, 2006). In cofilin mutants with reduced actin binding and severing activity, contractile rings failed to assemble normally from nodes, but instead assembled rings more slowly via diverse intermediate structures (Chen and Pollard, 2011). It was argued (Chen and Pollard, 2011) that cofilin-mediated actin filament severing is required for assembly from the nodes which are pulled together by pairwise actin filament connections that need regular renewal by severing to avoid node clumping and assembly failure (Vavylonis et al., 2008).

The role of cofilin during constriction of the assembled ring is much less clear. Is cofilin needed for constriction, and if so what is the mechanism? Fission yeast contractile rings with cofilin mutants constricted at normal mean rates, but with more variability (Chen and Pollard, 2011). However, during constriction rings exhibited remarkable structural irregularities, in which segments of the ring peeled away from the plasma membrane into straight segments we call bridges (Cheffings et al., 2019; Chen and Pollard, 2011). As bridging tended to occur in ring segments with lower Myo2 density, it was suggested the instability originated in a combination of reduced local Myo2-mediated actin anchoring and reduced local ring tension in the bridging region (Cheffings et al., 2019).

The contractile ring is a complex cellular machine of many components, so understanding the mechanisms of constriction and force production can benefit from mathematical modeling (Glotzer, 2005; O’Shaughnessy and Thiyagarajan, 2018; Pollard and O'Shaughnessy, 2019). Fission yeast is uniquely positioned for realistic quantitative modeling due to a wealth of available data on the identities, amounts, biochemical properties and organization of ring components (Chatterjee and Pollard, 2019; Chen et al., 2015; Courtemanche et al., 2016; Friend et al., 2018; Hayakawa et al., 2020; Pollard et al., 2017; Pollard and Wu, 2010a; Stark et al., 2010; Takaine et al., 2015; Wu and Pollard, 2005). This data was used to constrain models of the contractile ring which reproduced structural features and ring tensions measured in live cells (Alonso-Matilla et al., 2019; McDargh et al., 2021; Stachowiak et al., 2014; Thiyagarajan et al., 2017). In contrast to the classic sarcomeric force production 
mechanism of striated muscle (Huxley and Niedergerke, 1954; Huxley and Hanson, 1954), a sliding node mechanism emerged, where Myo2 and the unconventional myosin Myp2 (Bezanilla et al., 1997) pull nodes around the ring via attached actin filaments barbed-end-anchored to nodes via formins (McDargh et al., 2021). This generates tension in filaments, and a net ring tension. Experimental and modeling studies of septation, the growth of new cell wall in the wake of the constricting ring, suggest septation determines the constriction rate while the centripetal force from the ring mechanically regulates septation (Thiyagarajan et al., 2015; Zhou et al., 2015).

Here we used mathematical modeling to study the role of cofilin-mediated turnover in constricting contractile rings. Using a well-tested simulation framework (McDargh et al., 2021), we studied fission yeast contractile rings with the ADF-M3 cofilin mutant with reduced actin binding and severing activity (Chen and Pollard, 2011). Simulations quantitatively reproduced the experimentally observed bridges, the correlation with lower Myo2 densities and, remarkably, the cyclic character of bridging with $\sim 6$ min per bridging-rehealing episode (Cheffings et al., 2019). The origin of the bridging instability is that actin filaments grow much longer without cofilin-mediated severing, so the ring tension is massively boosted, sufficient to pull actin filaments away from the Myo2 that anchors them to the membrane. In live cells (Laplante et al., 2015) and in simulations (McDargh et al., 2021), essentially the same bridging instability is seen in myo2-E1 mutants with weakened binding to actin, showing that either increased centripetal loading or reduced anchoring provokes bridging. Thus, by maintaining actin filament lengths within a safe bound, cofilin protects the contractile ring from bridging instability, the principal structural threat to the contractile ring.

\section{Results}

\section{Molecularly detailed simulation of the fission yeast cytokinetic ring}

To examine the role of cofilin we adapted a molecularly explicit model of the fission yeast contractile ring we developed previously (McDargh et al., 2021), severely constrained by extensive data making the fission yeast contractile ring presently the most amenable to realistic mathematical modeling. The amounts of many ring components were measured over time and many were biochemically characterized (Chatterjee and Pollard, 2019; Chen et al., 2015; Courtemanche et al., 2016; Friend et al., 2018; Hayakawa et al., 2020; Pollard et al., 2017; Pollard and Wu, 2010b; Stark et al., 2010; Takaine et al., 2015; Wu and Pollard, 2005). Super-resolution FPALM microscopy showed the actomyosin organization in constricting rings is built from protein complexes called nodes (Laplante et al., 2016), anchored to the plasma membrane (PM). Each node contains myosin II Myo2, the actin filament nucleator formin Cdc12 and other components. A second unconventional myosin II Myp2 does not belong to the nodes and is likely unanchored from the plasma membrane.

Here we summarize the main points of the model (for more details see ref. (McDargh et al., 2021). Parameter values are listed in Material and Methods. In the model each node is a coarse- 
grained representation of the organization revealed by FPALM, with Myo2, formin Cdc12, IQGAP Rng2 and F-BAR protein Cdc15 (Laplante et al., 2016) (Fig. 1A). Formin Cdc12 dimers nucleate and grow actin filaments, binding the node $44 \mathrm{~nm}$ from the membrane, while the heads of $\sim 8$ Myo2 dimers are represented by an ellipsoid with dimensions and location matching experiment (132 x $102 \times 102 \mathrm{~nm}, 94 \mathrm{~nm}$ from the membrane). Semiflexible actin filaments are represented by chains of rods with bending stiffness corresponding to the $\sim 10 \mu \mathrm{m}$ persistence length (Ott et al., 1993), and Myp2 is represented as unanchored $200 \mathrm{~nm}$ clusters of 16 molecules each (Cheffings et al., 2019; Laplante et al., 2015; Takaine et al., 2015).

Filaments intersecting Myo2 or Myp2 clusters bind and are pulled (Fig. 1B), following linear force-velocity relations with total stall forces per cluster, $f_{\mathrm{Myo} 2}^{\text {stall }}$ and $f_{\mathrm{Myp} 2}^{\text {stall }}$, and with the measured load-free velocity $v_{m y o}^{0}$ for the estimated 25 myosin II heads per actin filament, $v_{m y o}^{0}=$ $0.24 \mu_{\mathrm{m} \mathrm{s}^{-1}}$ (Stark et al., 2010). Drag forces resist node membrane anchor motion with drag coefficient chosen to reproduce the experimental node velocity, $22 \pm 10 \mathrm{~nm} \mathrm{~s}^{-1}$ (Laplante et al., 2016). Steric forces oppose overlap of components, ensuring actin filaments do not cross. Actin filaments are dynamically crosslinked by Ain1 $\alpha$-actinin dimers (Wu and Pollard, 2005).

The component amounts follow the experimental values throughout constriction (Courtemanche et al., 2016; Wu and Pollard, 2005) (e.g., 3300 Myo2 molecules, 230 formin dimers in $\sim 210$ nodes, 2300 Myp2 molecules in $\sim 140$ clusters, and 230 actin filaments of total length $\sim 580 \mu \mathrm{m}$ at constriction onset). Nodes stochastically bind a $0.2 \mu \mathrm{m}$ wide zone of the ingrowing septum (Cortes et al., 2007), with mean dissociation time $41 \mathrm{~s}$ consistent with reported component dissociation times (Clifford et al., 2008; Pelham and Chang, 2002; Yonetani et al., 2008).

On binding a node a formin nucleates a randomly oriented actin filament. Cofilin-mediated severing stochastically shortens filaments homogenously with rate $r_{\mathrm{sev}}=0.93 \mu \mathrm{m}^{-1} \mathrm{~min}^{-1}$ (see Table S1) (Elam et al., 2013). Myp2 clusters bind actin filaments with turnover time 46 s (Takaine et al., 2015). Rates of severing, binding and actin polymerization are set by demanding simulations reproduce the experimental densities of Myo2, Myp2 and formin and mean actin length, all evolving as constriction progresses (Fig. 1C).

\section{Simulations reproduce forces and structural properties of experimental contractile rings}

We ran simulations of the model with a septum growing inwards at $2.4 \mathrm{~nm} \mathrm{~s}^{-1}$ (Thiyagarajan et al., 2015) for $\sim 25 \mathrm{~min}$. Components were initially randomly positioned in a $200 \mathrm{~nm}$ wide circular band representing the membrane lining the inside of the septum. Constriction was initialized after a preliminary $3 \mathrm{~min}$ run to allow the ring to self-organize. This dynamic organization maintained itself throughout constriction and reproduced many experimental features (Fig. 2A). Consistent with experiment, the actin filaments formed $\mathrm{a} \sim 130 \mathrm{~nm}$ thick bundle of $\sim 47$ filaments associated with a $100 \mathrm{~nm}$ thick Myo2 ring overlapping a concentric $200 \mathrm{~nm}$ thick inner Myp2 ring, separated by $26 \mathrm{~nm}$ (Laplante et al., 2015; Laplante et al., 2016; McDonald et al., 2017; Swulius et al., 2018). 
Ring tension was generated by a sliding node mechanism (Fig. 2B), very different to the classic sarcomeric mechanism of striated muscle (Huxley and Niedergerke, 1954; Huxley and Hanson, 1954). When incoming nodes bound the membrane, their formins nucleated actin filaments which were pulled by Myo2 belonging to other nodes. Since the membrane-anchored nodes host $\sim 1$ actin filament on average, they were pulled clockwise or counterclockwise around the ring depending on the actin orientation, with a node velocity distribution closely reproducing that measured using FPALM (Laplante et al., 2016; McDargh et al., 2021). In this way, filaments with barbed ends anchored to the membrane became tense, and a net ring tension was generated by the $\sim 200$ nodes (at constriction onset) (Fig. 2B). Unanchored Myp2 clusters also contributed by pulling filaments. Ring tensions increased from $\sim 500$ to $\sim 1000 \mathrm{pN}$ as rings constricted (Fig. 4C), with a mean of $\sim 740 \mathrm{pN}$, close to the values measured in live fission yeast protoplasts (McDargh et al., 2021).

\section{Bridging instabilities are the principal structural threat to the contractile ring}

The contractile ring builds a specialized organization from actin, myosin and other components that efficiently exerts centripetal force to drive constriction. For functionality, this organization must be stable over the $\sim 25$ mins of constriction. Our simulations and experiments show that the biggest structural threat originates in the high tensions in filaments that must bend to form the circular bundle at the core of the ring (McDargh et al., 2021). Energetically, bent tensile filaments prefer to straighten by pulling away from the membrane into straight bridges (Fig. 3A).

Now the centripetal force per length tending to straighten a segment of a ring of radius $R_{\text {ring }}$ and tension $T$ into a bridge is $T / R_{\text {ring }}$ (Fig. 3A). Further, the principal ring component anchoring actin to the membrane is the membrane-anchored Myo2, which binds actin filaments abundantly and supports them against radial displacement (McDargh et al., 2021). Myp2 makes a similar bundling contribution.

Thus we anticipate bridging will be precipitated by either (i) weakened actin-Myo2 binding, or (ii) increased ring tension $T$ (higher centripetal force). Accordingly we expect bridging in simulations (i) with the temperature-sensitive Myo2-E1 mutant, which binds actin weakly and has minimal ATPase activity (Lord and Pollard, 2004; Stark et al., 2013), and (ii) with artificially

elevated ring tensions $T$ by using unphysiologically higher values of $f_{\mathrm{Myo2}}^{\text {stall }}$ (4-fold increase), the forces exerted by Myo2 clusters (see Material and Methods). In both cases, rings suffered significant bridging, with Myp2-containing actin bridges $0.5-1.5 \mu \mathrm{m}$ in length (Fig. 3B, C). The bridging in simulated myo2-E1 rings (McDargh et al., 2021) closely matched bridging observed in live cells of the myo2-E1 mutant, a vivid demonstration of the instability (Laplante et al., 2015) (Fig. 3C).

\section{Contractile rings in adf1-M3 mutants with reduced actin severing have longer actin filaments and higher ring tension}

$\mathrm{ADF} /$ cofilin family proteins promote actin turnover by severing actin filaments (Andrianantoandro and Pollard, 2006; Elam et al., 2013; Pavlov et al., 2007). In adf1-M2 and adf1-M3 mutants, the 
severing activity is reduced but constriction rates are normal (Cheffings et al., 2019; Chen and Pollard, 2011). To study the effects of cofilin we ran simulations to mimic the adf1-M3 mutant. Adf1-M3 has two mutations (E132A, K133A), binds actin filaments 10-fold more slowly than wild-type and severs actin defectively (Chen and Pollard, 2011). In a severing assay (Chen and Pollard, 2011), Adf1-M3 required a 10-fold higher concentration than wild-type Adf1 for maximal actin severing activity, and even then the severing rate was $\sim 3$-fold reduced from wildtype. Thus to simulate adfl-M3 rings we set the severing rate to zero, $r_{\mathrm{sev}}=0$. Actin then dissociates only as whole filaments when formins dissociate from nodes (McDargh et al., 2021) (Fig. 1C).

In adf1-M3 simulations (Fig. 4A) actin filaments were $\sim 2$-fold longer than in wild-type simulations (Fig. 4B). Further, ring tension was $\sim 2$-fold increased over much of the course of constriction (Fig. 4C). Given reduced severing, longer actin filaments are expected, but the enhanced tension is less obvious. To understand this, we measured tensions in individual actin filaments and found that the maximal tension at the anchored barbed end increased roughly linearly with filament length in wild-type simulations (Fig. 4D). Thus, longer filaments have higher tension, because they are bound by more myosin-II dimers. Since the total ring tension is a sum of all filament contributions over the bundle cross-section (Fig. 2B), the net ring tension is higher with longer filaments (McDargh et al., 2021).

\section{Simulations reproduce bridging observed in contractile rings of adf1-M3 mutants}

Interestingly, bridging instabilities are seen for the adf1-M2 and adf1-M3 mutants (Cheffings et al., 2019) with reduced severing activity (Fig. 4A). Why reduced severing activity triggers bridging is unknown. Our simulation results above have already provided a strong clue: longer actin filaments lead to higher ring tension $T$ (Fig. 4B-D), and higher tension generates higher centripetal Laplace forces $\sim T / R_{\text {ring }}$ which promote bridging (Fig. 3A).

This line of reasoning was indeed confirmed by simulations, which showed dramatic bridging instabilities in cofilin mutants. The simulated bridges were remarkably similar to those observed in live cells (Fig. 4A). Leaving Myo2 behind, bridges pulled away from the membrane and comprised several actin filaments bundled by Myp2, as expected since Myp2 is unanchored and its localization to the ring depends on actin (Laplante et al., 2015; Takaine et al., 2015). Each bridge had a few tenuous attachments to the ring at the bridge ends, mediated by barbed-endanchored actin filaments.

\section{Bridge formation is negatively correlated with Myo2 concentration}

Consistent with experiment (Cheffings et al., 2019), bridging was more frequent in regions of lower Myo2 density around the ring. Since Myo2 is hosted by nodes, this was reflected by a negative correlation between node density and bridge location: the node density was $\sim 2$-fold lower in regions of the ring where a bridge occurred than in regions without a bridge ( $n=30$ rings, Fig. $5 \mathrm{~A})$. 
Interestingly, this same correlation is seen in experimental images of adf1-M3 contractile rings, where the Myo2 density is clearly lower in regions of the membrane from which a bridge has pulled away (Fig. 4A).

These observations in live cells and in simulations are consistent with bridging being a failure of Myo2-mediated anchoring. In this mechanism, tense actin filaments pull away from Myo2 that normally binds them to the membrane. Bridges thus peel away from the plasma membrane at locations where the inward Laplace forces exceed the local anchoring force from Myo2, which depends on the Myo2-actin unbinding threshold and the number of Myo2 heads that bind filaments. Thus, bridging is most likely in regions with low Myo2 density.

\section{Simulations reproduce cyclic bridging observed in contractile rings of cofilin mutants}

In adf1-M3 cells, bridging in contractile rings was cyclic with a period of $\sim 6 \mathrm{~min}$ and about 3 cycles over the course of ring constriction (Fig. 5B). Remarkably, our simulations quantitatively reproduced this behavior with considerable accuracy: $\sim 3$ cycles of bridging and self-assembly occurred during constriction, each lasting $\sim 6$ min (Fig. 5B).

We argue that the reproduction of this very specific experimental phenotype strongly suggests the simulation recapitulates the essential mechanisms governing the fission yeast contractile ring. In particular, we conclude that cofilin-mediated turnover that maintains actin filament lengths within a certain range are essential to protect the ring from bridging instability.

\section{Discussion}

Here we presented evidence that actin turnover is essential for structural stability of the actomyosin contractile ring. By regulating the length of actin filaments, ADF/cofilin-mediated severing protects the ring from bridging, its principal structural threat manifested in live cells in myo2-E1 (Laplante et al., 2015) and adf1 mutants (Cheffings et al., 2019; Chen and Pollard, 2011). Our modeling results explain why ring disruption due to this instability is expected in these two apparently unrelated contexts: in one case due to weakened anchoring, in the other due to increased unanchoring force.

The principal job of the contractile ring is to generate tension and exert inward centripetal force that directs constriction, accomplished by myosin II pulling on the bundled curved actin filaments in the ring and making them tense. However, a curved actin filament under tension has an enormous energetic preference to become straight. A typical filament has a length $l_{\text {act }}$ of the same order as its curvature, $1 / R_{\text {ring }}$, where $R_{\text {ring }} \sim 1 \mu \mathrm{m}$ is the radius of the ring. Thus its endto-end distance increases by $\Delta L \sim R_{\text {ring }}$ on straightening, giving an energy advantage $-\Delta F \sim T_{\text {fil }} \Delta L \sim 4000 k T$ where $T_{\text {fil }} \sim 15 \mathrm{pN}$ is a typical filament tension (McDargh et al., 2021). Summed over a bundle this yields an enormous thermodynamic driving force for actin filament bundles in the ring to straighten into bridges. Thus the contractile ring is vulnerable to straightening instability and must be stabilized by anchoring. We find the principal anchoring 
agent is the membrane-anchored Myo2 which binds actin filaments (McDargh et al., 2021) (see Fig. 3A, C).

The contractile ring has presumably evolved to efficiently generate tension $T$, given the available myosin II in the ring. However, above a certain level tension is structurally dangerous, as the force $\sim T / R_{\text {ring }}$ that powers bridging will exceed the opposing Myo2-mediated anchoring forces. Cofilin-mediated severing plays a critical role because the tension depends on the actin filament length, $T \sim \rho_{\text {myo }} l_{\text {act }}$ (McDargh et al., 2021). Thus, for a given density $\rho_{\text {myo }}$ of myosin II along the ring, longer filaments lead to higher ring tension and above a certain length provoke bridging. It is cofilin's job to prune the actin filaments sufficiently that their length never exceeds this dangerous threshold (Fig. 4B).

Actin turnover as a necessary stabilizing process to protect the structure of actomyosin contractile assemblies is an emerging theme. Component turnover was proposed to allow the fission yeast ring to rapidly reassemble itself without trauma as it gradually constricts (McDargh et al., 2021), and actin turnover is required for homeostasis of the ring (Chew et al., 2017). In Drosophila embryos, actomyosin assemblies driving apical constriction were observed to recoil from adherens junctions when cofilin was inhibited by depleting cofilin phosphatase (Jodoin et al., 2015). Following breakage, the mechanical connections between the actomyosin structures and the adherens junctions were re-established on a $\sim 2$ min timescale (Jodoin et al., 2015). This rupture-repair cycle is reminiscent of the $\sim 6 \mathrm{~min}$ bridging-repair cycles in the fission yeast contractile ring (Cheffings et al., 2019).

\section{Material and Methods}

We used our well-tested molecularly explicit model of the contractile ring (McDargh et al., 2021). Parameters used are shown in Table 1. For simulations with artificially higher Myo2 stall force

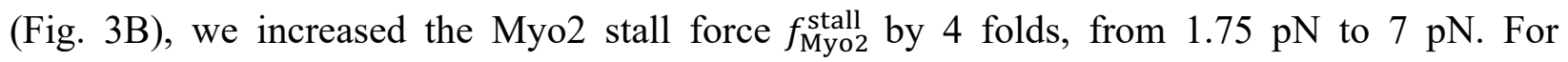
simulations of myo2-E1 mutants (Fig. 3C), we reduced the Myo2 stall force $f_{\mathrm{Myo} 2}^{\text {stall }}$ to zero, and lower the unbinding threshold $f_{\text {unbind }}^{\mathrm{Myo} 2}$ from $40 \mathrm{pN}$ to $12 \mathrm{pN}$ (McDargh et al., 2021). For simulations of adf1-M3 mutants, we reduced the severing rate $r_{\text {sev }}$ to zero. For other details of our molecularly explicit model, please see Ref (McDargh et al., 2021).

Table 1. Key parameter values of the ring simulation.

\begin{tabular}{|l|l|l|l|}
\hline Symbol & Meaning & $\begin{array}{l}\text { Value at onset } \\
\text { of constriction }\end{array}$ & Legend \\
\hline & Ring binding zone width & $0.2 \mu \mathrm{m}$ & (A) \\
\hline & Initial ring length & $11.6 \mu \mathrm{m}$ & (B) \\
\hline & Ring constriction rate & $70 \mathrm{~nm} \mathrm{~min}^{-1}$ & (B) \\
\hline
\end{tabular}




\begin{tabular}{|c|c|c|c|}
\hline$\rho_{\mathrm{Cdc} 12 \mathrm{p}}$ & $\begin{array}{l}\text { Density of formin Cdc12p dimers along } \\
\text { the ring }\end{array}$ & $20 \mu \mathrm{m}^{-1}$ & $(\mathrm{C})^{*}$ \\
\hline$\rho_{\text {Myo2 }}$ & Density of Myo2 nodes along the ring & $18 \mu \mathrm{m}^{-1}$ & (D) ${ }^{*}$ \\
\hline$\rho_{\text {Myp2 }}$ & Density of Myp2 clusters along the ring & $12.5 \mu \mathrm{m}^{-1}$ & $(\mathbf{E})^{*}$ \\
\hline$k_{\text {Myo2 }}^{\text {off }}$ & Myo2 off rate & $0.0245 \mathrm{~s}^{-1}$ & (F) \\
\hline$k_{\mathrm{Myp} 2}^{\text {off }}$ & Myp2 off rate & $0.026 \mathrm{~s}^{-1}$ & (G) \\
\hline$f_{\text {Myo2 }}^{\text {stall }}$ & Myo2 stall force per head & $1.75 \mathrm{pN}$ & (H) \\
\hline \multirow[t]{4}{*}{$f_{\text {Myp2 }}^{\text {stall }}$} & Myp2 stall force per head & $1.0 \mathrm{pN}$ & $(\mathbf{H})$ \\
\hline & Major axis of Myo2 capture zone & $132 \mathrm{~nm}$ & (I) \\
\hline & Minor axes of Myo2 capture zone & $102 \mathrm{~nm}$ (both) & (I) \\
\hline & Diameter of Myp2 capture zone & $200 \mathrm{~nm}$ & (J) \\
\hline$\rho_{\text {actinin }}$ & $\begin{array}{l}\text { Density of } \alpha \text {-actinin dimers along the } \\
\text { ring }\end{array}$ & $25 \mu \mathrm{m}^{-1}$ & $(\mathbf{K})$ \\
\hline$k_{\text {actinin }}^{\text {off }}$ & $\alpha$-actinin off rate & $3.3 \mathrm{~s}^{-1}$ & (L) \\
\hline$r_{\mathrm{sev}}$ & $\begin{array}{l}\text { Cofilin-mediated severing rate per unit } \\
\text { length on actin filament }\end{array}$ & $0.93 \mu \mathrm{m}^{-1} \mathrm{~min}^{-1}$ & $(\mathbf{M})^{*}$ \\
\hline$v_{\text {pol }}$ & $\begin{array}{l}\text { Formin-mediated actin polymerization } \\
\text { rate }\end{array}$ & $127 \mathrm{~nm} \mathrm{~s}^{-1}$ & $(\mathbf{M})^{*}$ \\
\hline$l_{\mathrm{p}}$ & Actin filament persistence length & $10 \mu \mathrm{m}$ & $(\mathbf{N})$ \\
\hline$\gamma_{\text {anch }}$ & Anchor drag coefficient per node & $500 \mathrm{pN} \cdot \mathrm{s} \mu \mathrm{m}^{-1}$ & (O) \\
\hline$k_{\mathrm{ex}}^{\text {Myp2-Myp2 }}$ & $\begin{array}{l}\text { Myp2-Myp2 excluded volume spring } \\
\text { constant }\end{array}$ & $0.32 \mathrm{pN} \mathrm{nm}^{-1}$ & \\
\hline$k_{\mathrm{ex}}^{\mathrm{Myo2-Myo2}}$ & $\begin{array}{l}\text { Myo2-Myo2 excluded volume spring } \\
\text { constant }\end{array}$ & $0.12 \mathrm{pN} \mathrm{nm}^{-1}$ & \\
\hline$k_{\mathrm{ex}}^{\text {Anchor-Myp2 }}$ & $\begin{array}{l}\text { Myo2-Myo2 excluded volume spring } \\
\text { constant }\end{array}$ & $0.095 \mathrm{pN} \mathrm{nm}^{-1}$ & \\
\hline$r_{0}^{\text {Myp2-Myp2 }}$ & $\begin{array}{l}\text { Myp2-Myp2 excluded volume cutoff } \\
\text { distance }\end{array}$ & $200 \mathrm{~nm}$ & \\
\hline
\end{tabular}




\begin{tabular}{|c|c|c|c|}
\hline$r_{0}^{\text {Муо2-Муо2 }}$ & $\begin{array}{l}\text { Myo2-Myo2 excluded volume cutoff } \\
\text { distance }\end{array}$ & $132 \mathrm{~nm}$ & \\
\hline$r_{0}^{\text {Anchor-Myp2 }}$ & $\begin{array}{l}\text { Anchor-Myp2 excluded volume cutoff } \\
\text { distance }\end{array}$ & $202 \mathrm{~nm}$ & \\
\hline$f_{\text {act }}$ & $\begin{array}{l}\text { Actin-Actin excluded volume } \\
\text { maximum force }\end{array}$ & $10 \mathrm{pN}$ & \\
\hline$r_{\mathrm{ex}}$ & Actin-Actin excluded volume distance & $15 \mathrm{~nm}$ & \\
\hline$f_{\text {filament }}$ & Actin-limited stall force & $8 \mathrm{pN}$ & (P) \\
\hline$f_{\text {unbind }}^{\text {Myo2 }}$ & Myo2-actin unbinding threshold force & $40 \mathrm{pN}$ & (Q) \\
\hline$f_{\text {unbind }}^{\text {Myp2 }}$ & Myp2-actin unbinding threshold force & $30 \mathrm{pN}$ & (Q) \\
\hline$v_{\text {myo }}^{0}$ & Load-free Myo2/Myp2 velocity & $0.24 \mu \mathrm{m} \mathrm{s}^{-1}$ & (R) \\
\hline$k_{\mathrm{x}}$ & Crosslinker spring constant & $25 \mathrm{pN} \mu \mathrm{m}^{-1}$ & (S) \\
\hline$r_{\mathrm{x}}$ & Crosslinker rest length & $30 \mathrm{~nm}$ & (S) \\
\hline$\theta_{\max }$ & $\begin{array}{l}\text { Maximum angle between new actin } \\
\text { filament and ring }\end{array}$ & $30^{\circ}$ & (T) \\
\hline
\end{tabular}

\section{Legend:}

* indicates that these parameters were tuned throughout constriction to ensure the time course of component numbers matched previous experimental measurements.

(A) We assume that new constriction nodes bind the leading edge of the septum (in-growing cell wall). The septum has width $\sim 0.2 \mu \mathrm{m}$ (Cortes et al., 2007).

(B) Measured in (Pelham and Chang, 2002).

(C) 200 dimers of Cdc12 were measured at constriction onset for a ring with circumference $\sim 10 \mu \mathrm{m}$. (Courtemanche et al., 2016). For simplicity we neglect formin For3 that is also present, since For3 grows actin cables (Feierbach and Chang, 2001) and 4 for 3 cells have normal actin levels in the ring and lack observable cytokinesis defects.

(C) (Yonetani et al., 2008). Together with the value for $\rho_{\text {Cdc12 }}$, this gives a formin binding rate of $r_{\text {Cdc12p }}=$ $0.46 \mu \mathrm{m}^{-1} \cdot \mathrm{s}^{-1}$ at the onset of constriction.

(D) Calculated from 2900 Myo2p in a $10 \mu \mathrm{m}$ long ring at the onset of constriction (Wu and Pollard, 2005), and 8 Myo2 dimers per node (Laplante et al., 2016).

(E) Calculated from 2000 Myp2p in a $10 \mu \mathrm{m}$ long ring at the onset of constriction (Wu and Pollard, 2005), and 16 Myp2 heads per cluster, determined in this study (see STAR Methods).

(F) Lifetimes of myosin light chains Cdc4 (Pelham and Chang, 2002) and Rlc1 (Clifford et al., 2008) and the formin Cdc12 (Yonetani et al., 2008) were measured previously using FRAP. We calculated the off- 
rate from the Rlc1 lifetime and used it for the Myo2 off rate as this was in the middle of the range of experimental values.

(G) Consistent with off-rates of $0.022 \mathrm{~s}^{-1}$ obtained using previous FRAP measurements of Myp2 (Takaine et al., 2015).

(H) Fitted in (McDargh et al., 2021).

(I) From the distribution of Myo2 heads measured in FPALM (Laplante et al., 2016).

(J) From the apparent size of Myp2 clusters measured in deconvolution microscopy (Takaine et al., 2015).

(K) (Wu and Pollard, 2005).

(L) (Li et al., 2016).

(M) Fitted in (McDargh et al., 2021).

(N) (Ott et al., 1993; Riveline et al., 1997).

(O) Fitted in (McDargh et al., 2021) to reproduced the node velocity $\left(22 \pm 10 \mathrm{~nm} \mathrm{~s}^{-1}\right)$ measured by FPALM (Laplante et al., 2016).

(P) (Vavylonis et al., 2008)

(Q) Fitted in (McDargh et al., 2021).

(R) Calculated in (McDargh et al., 2021).

(S) Estimated from in vitro measurements on crosslinked actin bundles (Claessens et al., 2006) and from measurements of $\alpha$-actinin length using electron microscopy (Meyer and Aebi, 1990).

(T) Chosen to minimize the fraction of actin in whiskers. The overall structure of the ring was qualitatively insensitive to increases in this parameter, though the whisker fraction increases with larger maximum angles.

\section{Acknowledgements}

We thank Mohan Balasubramanian, University of Warwick, for providing experimental images and data of adfl-M3 rings for comparison. We thank Thomas D. Pollard, Yale University, for providing experimental images of myo2-E1 rings for comparison. We thank the High-Performance Computing clusters provided by Columbia University.

\section{Competing interests}

The authors declare no competing or financial interests.

\section{Funding}

This work was supported by National Institute of General Medical Sciences of the national Institutes of Health under award number R01GM086731 to B.O's. The content is solely the 
responsibility of the authors and does not necessarily represent the official views of the National Institutes of Health.

\section{Author Contributions}

B.O. conceived the study; Z.M. designed the model; Z.M., T.Z. and H.Z., performed simulations and analysis; B.O. wrote the paper.

\section{Data Availability}

All codes (MATLAB) of our molecular explicit model are available on our public GitHub page (https://github.com/OShaughnessyGroup-Columbia-

University/constriction_of_the_fission_yeast_contractile_ring). 


\section{References}

Abe, H., Obinata, T., Minamide, L.S., and Bamburg, J.R. (1996). Xenopus laevis actin-depolymerizing factor/cofilin: a phosphorylation-regulated protein essential for development. The Journal of cell biology 132, 871-885.

Alonso-Matilla, R., Thiyagarajan, S., and O'Shaughnessy, B. (2019). Sliding filament and fixed filament mechanisms contribute to ring tension in the cytokinetic contractile ring. Cytoskeleton 76, 611-625. Andrianantoandro, E., and Pollard, T.D. (2006). Mechanism of actin filament turnover by severing and nucleation at different concentrations of ADF/cofilin. Mol Cell 24, 13-23.

Bezanilla, M., Forsburg, S.L., and Pollard, T.D. (1997). Identification of a second myosin-II in Schizosaccharomyces pombe: Myp2p is conditionally required for cytokinesis. Molecular biology of the cell 8, 2693-2705.

Chatterjee, M., and Pollard, T.D. (2019). The Functionally Important N-Terminal Half of Fission Yeast Mid1p Anillin Is Intrinsically Disordered and Undergoes Phase Separation. Biochemistry 58, 3031-3041. Cheffings, T.H., Burroughs, N.J., and Balasubramanian, M.K. (2019). Actin turnover ensures uniform tension distribution during cytokinetic actomyosin ring contraction. Mol Biol Cell 30, 933-941. Chen, Q., Courtemanche, N., and Pollard, T.D. (2015). Aip1 promotes actin filament severing by cofilin and regulates constriction of the cytokinetic contractile ring. J Biol Chem 290, 2289-2300.

Chen, Q., and Pollard, T.D. (2011). Actin filament severing by cofilin is more important for assembly than constriction of the cytokinetic contractile ring. Journal of Cell Biology 195, 485-498.

Chew, T.G., Huang, J., Palani, S., Sommese, R., Kamnev, A., Hatano, T., Gu, Y., Oliferenko, S., Sivaramakrishnan, S., and Balasubramanian, M.K. (2017). Actin turnover maintains actin filament homeostasis during cytokinetic ring contraction. Journal of Cell Biology 216, 2657-2667.

Claessens, M.M., Bathe, M., Frey, E., and Bausch, A.R. (2006). Actin-binding proteins sensitively mediate F-actin bundle stiffness. Nat Mater 5, 748-753.

Clifford, D.M., Wolfe, B.A., Roberts-Galbraith, R.H., McDonald, W.H., Yates III, J.R., and Gould, K.L. (2008). The Clp1/Cdc14 phosphatase contributes to the robustness of cytokinesis by association with anillin-related Mid1. The Journal of cell biology 181, 79-88.

Cortes, J.C., Konomi, M., Martins, I.M., Munoz, J., Moreno, M.B., Osumi, M., Duran, A., and Ribas, J.C. (2007). The (1,3)beta-D-glucan synthase subunit Bgs1p is responsible for the fission yeast primary septum formation. Mol Microbiol 65, 201-217.

Courtemanche, N., Pollard, T.D., and Chen, Q. (2016). Avoiding artefacts when counting polymerized actin in live cells with LifeAct fused to fluorescent proteins. Nat Cell Biol 18, 676-683.

D’Avino, P.P., Giansanti, M.G., and Petronczki, M. (2015). Cytokinesis in Animal Cells. Cold Spring Harb Perspect Biol 7, a015834.

Elam, W.A., Kang, H., and De la Cruz, E.M. (2013). Biophysics of actin filament severing by cofilin. FEBS Lett 587, 1215-1219.

Feierbach, B., and Chang, F. (2001). Roles of the fission yeast formin for3p in cell polarity, actin cable formation and symmetric cell division. Curr Biol 11, 1656-1665.

Friend, J.E., Sayyad, W.A., Arasada, R., McCormick, C.D., Heuser, J.E., and Pollard, T.D. (2018). Fission yeast Myo2: Molecular organization and diffusion in the cytoplasm. Cytoskeleton (Hoboken) 75, 164173.

Glotzer, M. (2005). The Molecular Requirements for Cytokinesis. Science 307, 1735-1739.

Gunsalus, K.C., Bonaccorsi, S., Williams, E., Verni, F., Gatti, M., and Goldberg, M.L. (1995). Mutations in twinstar, a Drosophila gene encoding a cofilin/ADF homologue, result in defects in centrosome migration and cytokinesis. The Journal of cell biology 131, 1243-1259. 
Hayakawa, Y., Takaine, M., Imai, T., Yamada, M.D., Hirose, K., Ngo, K.X., Kodera, N., Tokuraku, K., Numata, O., Nakano, K., et al. (2020). Actin binding domain of Rng2 strongly inhibits actin movement on myosin II HMM through structural changes of actin filaments. bioRxiv, 2020.2004.2014.041046. Huxley, A.F., and Niedergerke, R. (1954). Structural Changes in Muscle During Contraction: Interference Microscopy of Living Muscle Fibres. Nature 173, 971-973.

Huxley, H., and Hanson, J. (1954). Changes in the Cross-Striations of Muscle during Contraction and Stretch and their Structural Interpretation. Nature 173, 973-976. Jodoin, Jeanne N., Coravos, Jonathan S., Chanet, S., Vasquez, Claudia G., Tworoger, M., Kingston, Elena R., Perkins, Lizabeth A., Perrimon, N., and Martin, Adam C. (2015). Stable Force Balance between Epithelial Cells Arises from F-Actin Turnover. Developmental Cell 35, 685-697.

Laplante, C., Berro, J., Karatekin, E., Hernandez-Leyva, A., Lee, R., and Pollard, T.D. (2015). Three Myosins Contribute Uniquely to the Assembly and Constriction of the Fission Yeast Cytokinetic Contractile Ring. Curr Biol 25, 1955-1965.

Laplante, C., Huang, F., Tebbs, I.R., Bewersdorf, J., and Pollard, T.D. (2016). Molecular organization of cytokinesis nodes and contractile rings by super-resolution fluorescence microscopy of live fission yeast. Proc Natl Acad Sci U S A 113, E5876-E5885.

Li, Y.J., Christensen, J.R., Homa, K.E., Hocky, G.M., Fok, A., Sees, J.A., Voth, G.A., and Kovar, D.R. (2016). The F-actin bundler alpha-actinin Ain1 is tailored for ring assembly and constriction during cytokinesis in fission yeast. Molecular Biology of the Cell 27, 1821-1833.

Lord, M., and Pollard, T.D. (2004). UCS protein Rng3p activates actin filament gliding by fission yeast myosin-II. J Cell Biol 167, 315-325.

McDargh, Z., Wang, S., Chin, H.F., Thiyagarajan, S., Karatekin, E., Pollard, T.D., and O'Shaughnessy, B. (2021). Myosins generate contractile force and maintain organization in the cytokinetic contractile ring. bioRxiv, 2021.2005.2002.442363.

McDonald, N.A., Lind, A.L., Smith, S.E., Li, R., and Gould, K.L. (2017). Nanoscale architecture of the Schizosaccharomyces pombe contractile ring. Elife 6.

Meyer, R.K., and Aebi, U. (1990). Bundling of actin filaments by alpha-actinin depends on its molecular length. J Cell Biol 110, 2013-2024.

Nakano, K., and Mabuchi, I. (2006). Actin-depolymerizing protein Adf1 is required for formation and maintenance of the contractile ring during cytokinesis in fission yeast. Molecular biology of the cell 17, 1933-1945.

O'Shaughnessy, B., and Thiyagarajan, S. (2018). Mechanisms of contractile ring tension production and constriction. Biophys Rev 10, 1667-1681.

Ono, K., Parast, M., Alberico, C., Benian, G.M., and Ono, S. (2003). Specific requirement for two ADF/cofilin isoforms in distinct actin-dependent processes in Caenorhabditis elegans. Journal of cell science 116, 2073-2085.

Ott, A., Magnasco, M., Simon, A., and Libchaber, A. (1993). Measurement of the persistence length of polymerized actin using fluorescence microscopy. Phys Rev E Stat Phys Plasmas Fluids Relat Interdiscip Topics 48, R1642-R1645.

Pavlov, D., Muhlrad, A., Cooper, J., Wear, M., and Reisler, E. (2007). Actin filament severing by cofilin. J Mol Biol 365, 1350-1358.

Pelham, R.J., and Chang, F. (2002). Actin dynamics in the contractile ring during cytokinesis in fission yeast. Nature $419,82-86$.

Pollard, L.W., Bookwalter, C.S., Tang, Q., Krementsova, E.B., Trybus, K.M., and Lowey, S. (2017). Fission yeast myosin Myo2 is down-regulated in actin affinity by light chain phosphorylation. Proc Natl Acad Sci U S A 114, E7236-E7244.

Pollard, T.D. (2007). Regulation of actin filament assembly by Arp2/3 complex and formins. Annu Rev Biophys Biomol Struct 36, 451-477. 
Pollard, T.D., and O'Shaughnessy, B. (2019). Molecular Mechanism of Cytokinesis. Annu Rev Biochem 88, 661-689.

Pollard, T.D., and Wu, J.-Q. (2010a). Understanding cytokinesis: lessons from fission yeast. Nature Reviews Molecular Cell Biology 11, 149-155.

Pollard, T.D., and Wu, J.Q. (2010b). Understanding cytokinesis: lessons from fission yeast. Nat Rev Mol

Cell Biol 11, 149-155.

Riveline, D., Wiggins, C.H., Goldstein, R.E., and Ott, A. (1997). Elastohydrodynamic study of actin filaments using fluorescence microscopy. Phys Rev E 56, R1330-R1333.

Stachowiak, M.R., Laplante, C., Chin, H.F., Guirao, B., Karatekin, E., Pollard, T.D., and O'Shaughnessy, B. (2014). Mechanism of cytokinetic contractile ring constriction in fission yeast. Dev Cell 29, 547-561.

Stark, B.C., James, M.L., Pollard, L.W., Sirotkin, V., and Lord, M. (2013). UCS protein Rng3p is essential for myosin-II motor activity during cytokinesis in fission yeast. PLoS One 8, e79593.

Stark, B.C., Sladewski, T.E., Pollard, L.W., and Lord, M. (2010). Tropomyosin and myosin-II cellular levels promote actomyosin ring assembly in fission yeast. Mol Biol Cell 21, 989-1000.

Swulius, M.T., Nguyen, L.T., Ladinsky, M.S., Ortega, D.R., Aich, S., Mishra, M., and Jensen, G.J. (2018). Structure of the fission yeast actomyosin ring during constriction. Proc Natl Acad Sci U S A 115, E1455E1464.

Takaine, M., Numata, O., and Nakano, K. (2015). An actin-myosin-Il interaction is involved in maintaining the contractile ring in fission yeast. J Cell Sci 128, 2903-2918.

Thiyagarajan, S., Munteanu, E.L., Arasada, R., Pollard, T.D., and O'Shaughnessy, B. (2015). The fission yeast cytokinetic contractile ring regulates septum shape and closure. J Cell Sci 128, 3672-3681. Thiyagarajan, S., Wang, S., and O'Shaughnessy, B. (2017). A node organization in the actomyosin contractile ring generates tension and aids stability. Molecular Biology of the Cell 28, 3286-3297.

Vavylonis, D., Wu, J.Q., Hao, S., O'Shaughnessy, B., and Pollard, T.D. (2008). Assembly mechanism of the contractile ring for cytokinesis by fission yeast. Science 319, 97-100.

Wu, J.-Q., Sirotkin, V., Kovar, D.R., Lord, M., Beltzner, C.C., Kuhn, J.R., and Pollard, T.D. (2006). Assembly of the cytokinetic contractile ring from a broad band of nodes in fission yeast. The Journal of cell biology 174, 391-402.

Wu, J.Q., and Pollard, T.D. (2005). Counting cytokinesis proteins globally and locally in fission yeast. Science 310, 310-314.

Yonetani, A., Lustig, R.J., Moseley, J.B., Takeda, T., Goode, B.L., and Chang, F. (2008). Regulation and targeting of the fission yeast formin cdc12p in cytokinesis. Molecular biology of the cell 19, 2208-2219. Zhou, Z., Munteanu, E.L., He, J., Ursell, T., Bathe, M., Huang, K.C., and Chang, F. (2015). The contractile ring coordinates curvature-dependent septum assembly during fission yeast cytokinesis. Molecular biology of the cell $26,78-90$. 
A
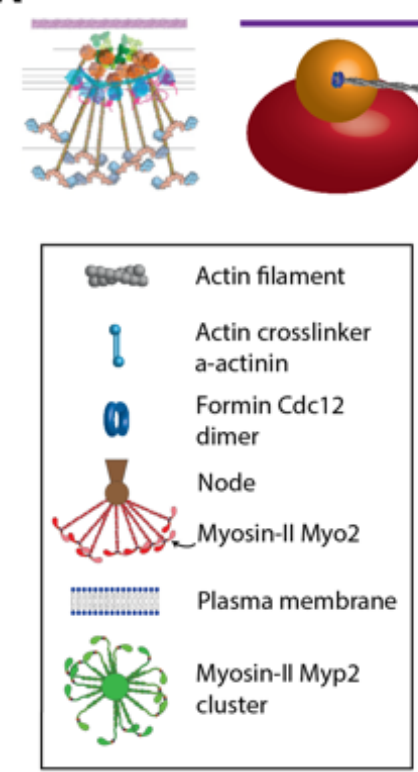

C

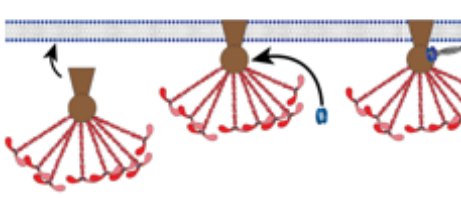

B

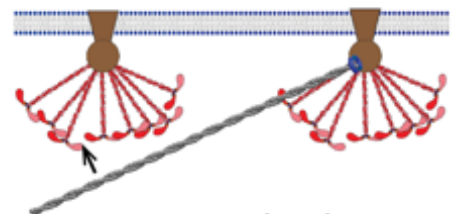

Myo2-actin binding

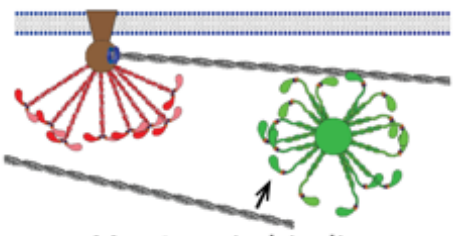

Myp2-actin binding

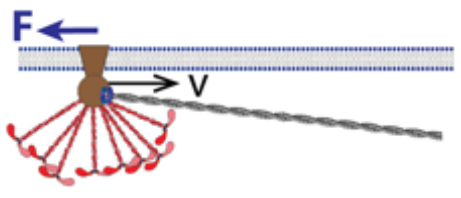

Anchor drag
Forces

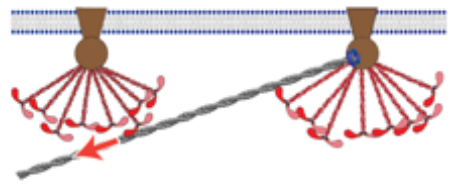

Myo2-actin pulling

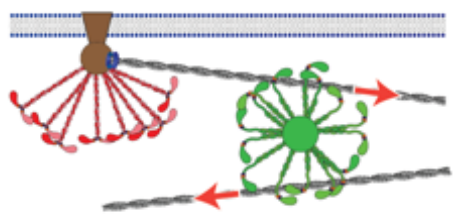

Myp2-actin pulling

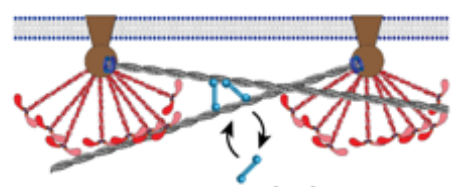

Actin crosslinking

Turnover

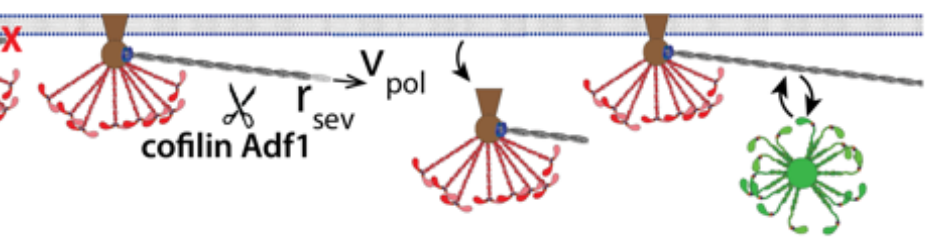

Figure 1. Molecularly explicit model of the $S$. pombe contractile ring. (A) Left: schematic of membrane-anchored node organization. Right: the coarse-grained model representation of myosin Myo2 (red) and formin Cdc15 and IQGAP Rng2 (orange). (B) Forces in the model. (C) Turnover of the ring components. Nodes bind and unbind to the plasma membrane. Formin Cdc15 dimers bind to nodes and polymerize randomly oriented actin filaments at rate $v_{\text {pol }}$. Cofilin Adf1 (the scissors) stochastically severs filaments with rate $r_{\text {sev }}$. Myp2 clusters bind and unbind actin filaments. 

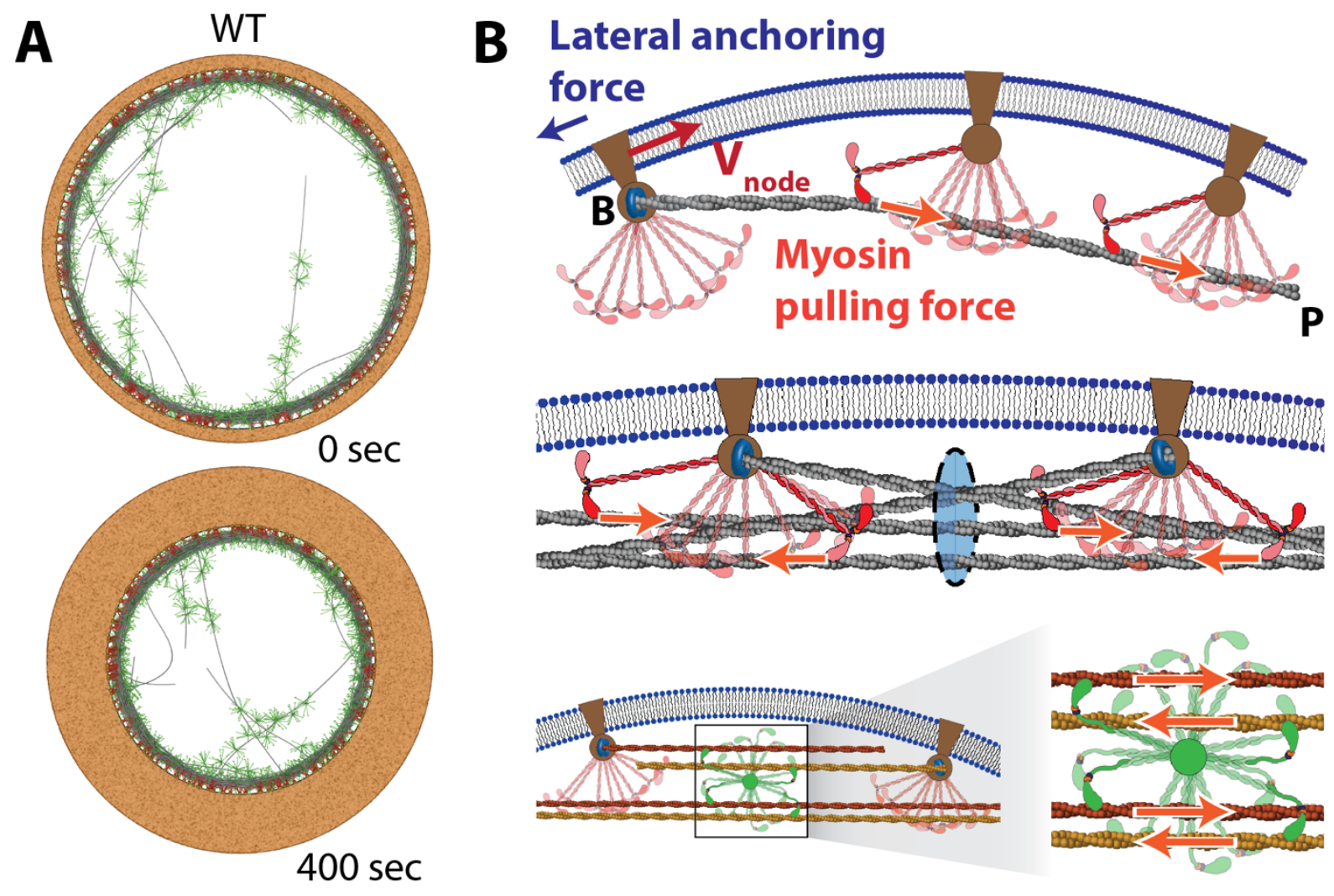

Figure 2. The constriction of a wild-type contractile ring and the sliding node ring tension mechanism. (A) The simulated wild-type contractile ring at different time steps (cross-section). The septum is represented by the orange edge around the ring. (B) The sliding node ring tension mechanism. Up: an actin filament is pulled by Myo2 heads and Myp2 heads (not shown) and the anchored barbed end provides drag force, which make the filament tense. Middle: the sum of the tensions of all the actin filaments passing through a given cross-section of the ring gives the ring tension. Bottom: unanchored Myp2 clusters contribute to ring tension by pulling on roughly equal numbers of oppositely oriented actin filaments. 
A

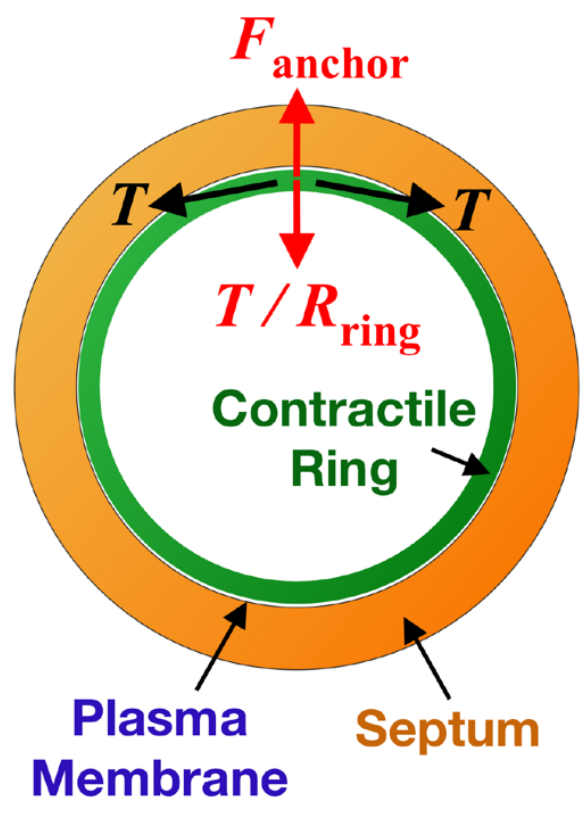

B
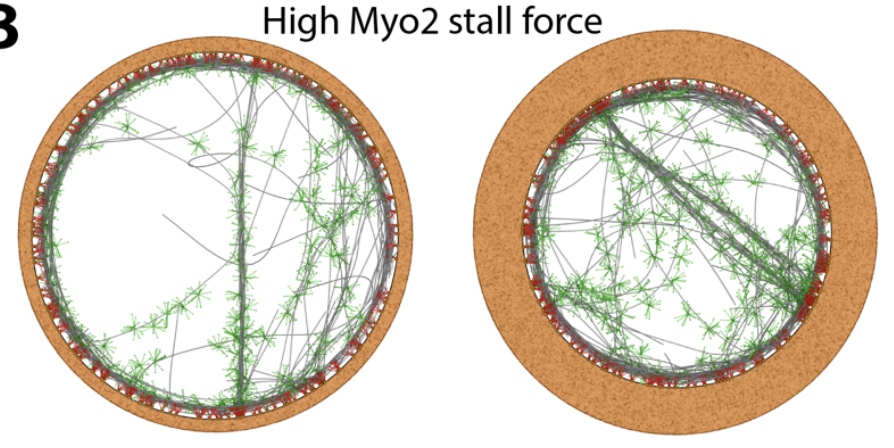

C

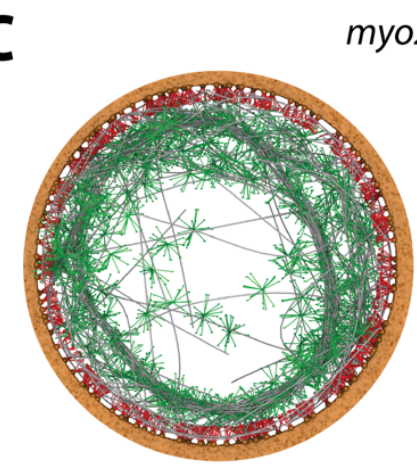

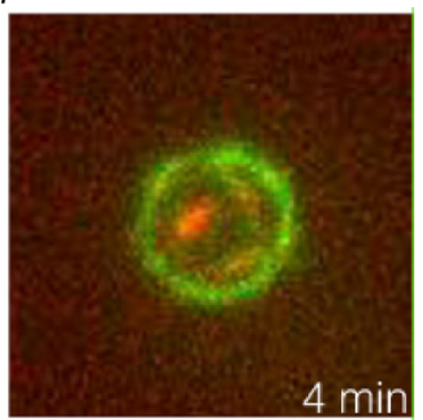

mCherry-Myp2p

GFP-CHD (actin)

Figure 3. The mechanism of bridging instabilities. (A) The centripetal force per length $T / R_{\text {ring }}$ results from the tension $T$ along a contractile ring of radius $R_{\text {ring }}$. Meanwhile, the membrane-anchored Myo2 provides anchoring force $F_{\text {anchor }}$ that prevents radial displacement of the ring (bridging). (B) Simulations with artificially quadrupled Myo2 stall force $f_{\text {Myo2 }}^{\text {stall }}$ show significant bridging. (C) Simulated (McDargh et al., 2021) and experimental (Laplante et al., 2015) myo2-E1 rings where Myo2 heads bind actin weakly show significant bridging. 
A

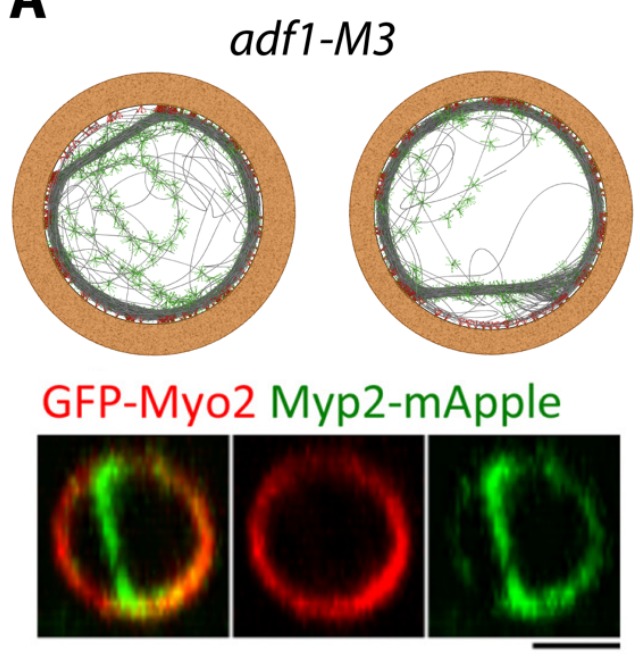

C

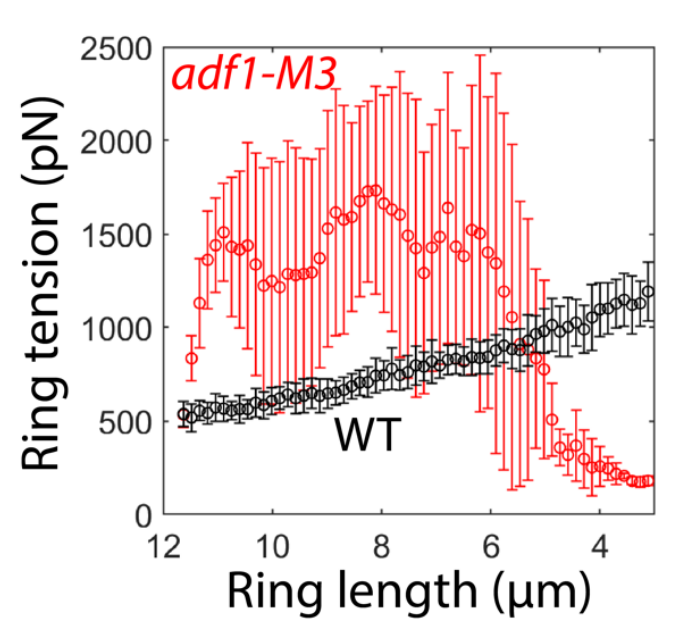

B

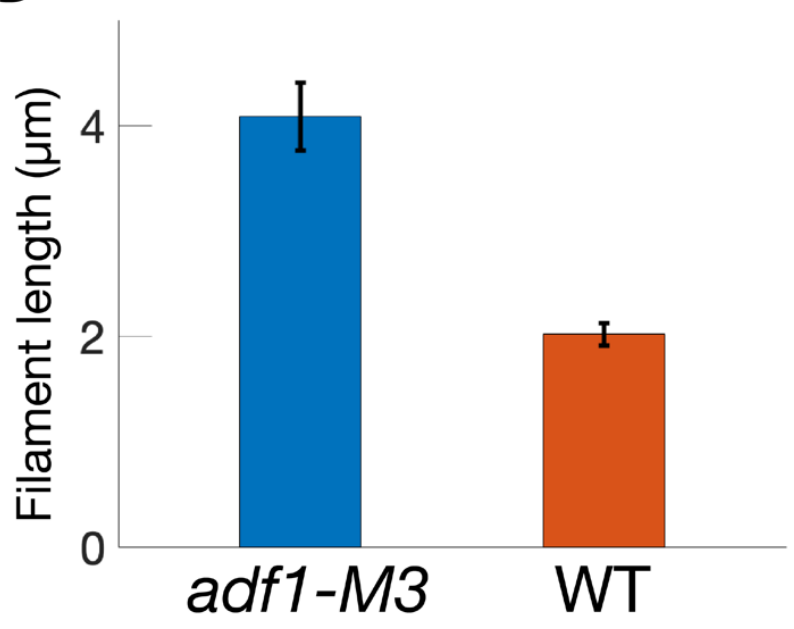

D

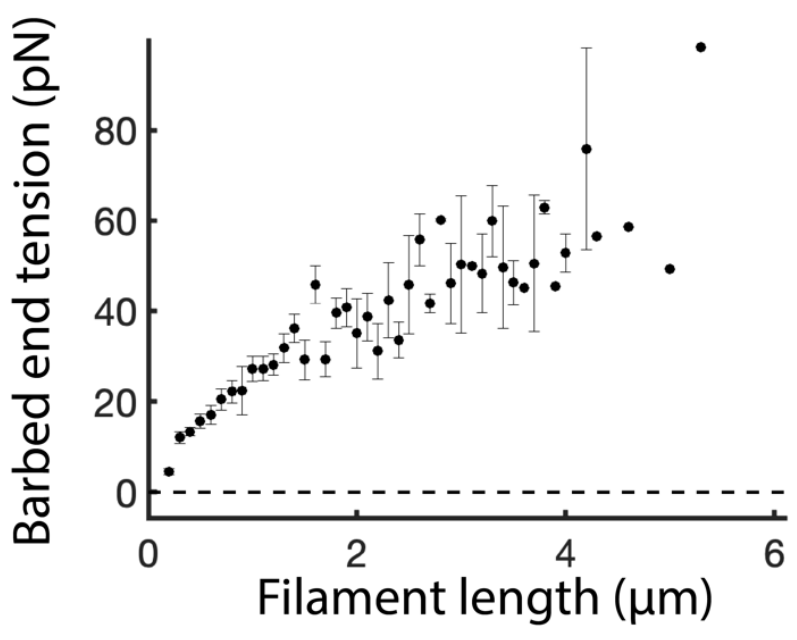

Figure 4. Cofilin mutant adf1-M3 leads to bridging because longer actin filaments give higher ring tension. (A) Simulated adf1-M3 rings with no actin severing reproduce the bridging observed experimentally (Cheffings et al., 2019). Both simulated and experimental bridges have accumulated Myp2 clusters. Scale bar: $2 \mu \mathrm{m}$. (B) Actin filament length (mean $\pm \mathrm{SD}$ ) in simulated adf1-M3 $(n=30)$ and wild-type $(n=60)$ rings. (C) Ring tension (mean \pm SD) during constriction in simulated adf1-M3 $(n=30)$ and wild-type $(n=60)$ rings. (D) Actin filament tension (mean $\pm \mathrm{SD}$ ) at the barbed ends vs. filament length in simulated wild-type rings ( $n=$ $60)$. 


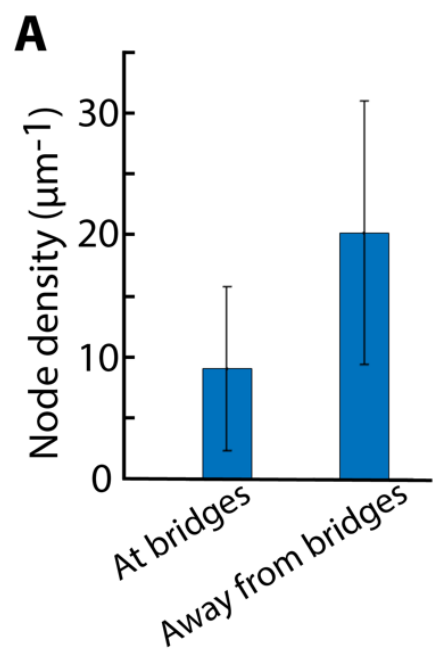

B

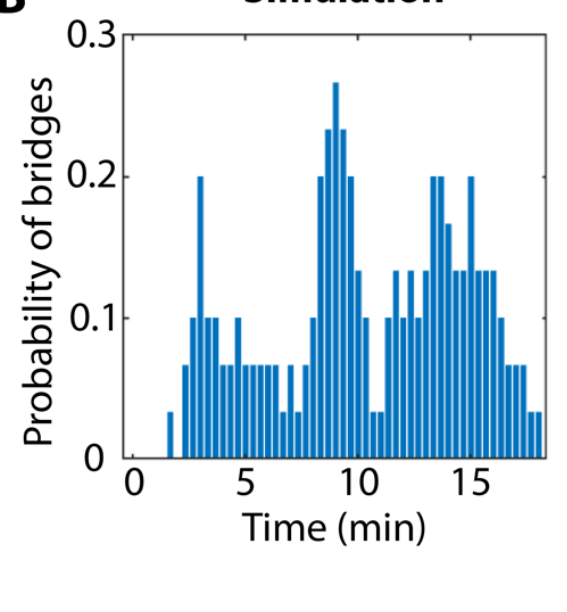

Experiment

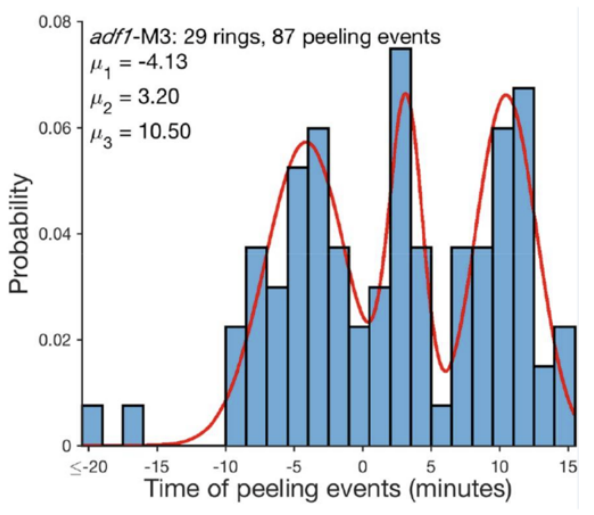

Figure 5. Simulated adf1-M3 rings show negative correlation of bridging with local Myo2 concentration and cyclic bridging. (A) Node density (mean \pm SD) at and away from bridging sites in simulated adf1-M3 rings. (B) Probability of bridges during constriction in simulated adf1-M3 rings $(n=30)$ (left) and experiment (Cheffings et al., 2019) (right). 